\title{
Les applaudissements comme source de tension : Étude de la réception des 24 Préludes de François Dompierre lors de leur création au Festival de Lanaudière Applause as a source of tension: A study of the reception of 24 Préludes by François Dompierre in the context of their premiere at the Festival de Lanaudière
}

\section{Danick Trottier}

Volume 14, numéro 2, automne 2013

La passion de la recherche (à la mémoire de Maryvonne Kendergian)

URI : https://id.erudit.org/iderudit/1023740ar

DOI : https://doi.org/10.7202/1023740ar

\section{Aller au sommaire du numéro}

\section{Éditeur(s)}

Société québécoise de recherche en musique

\section{ISSN}

1480-1132 (imprimé)

1929-7394 (numérique)

\section{Découvrir la revue}

Citer cet article

Trottier, D. (2013). Les applaudissements comme source de tension : Étude de la réception des 24 Préludes de François Dompierre lors de leur création au Festival de Lanaudière. Les Cahiers de la Société québécoise de recherche en musique, 14(2), 59-68. https://doi.org/10.7202/1023740ar

\section{Résumé de l'article}

La présente étude porte sur la réception des 24 Préludes de François Dompierre créés par Alain Lefèvre le 14 juillet 2012 à l'Amphithéâtre Fernand-Lindsay du Festival de Lanaudière. La soirée, placée sous le signe de la découverte d'une nouvelle oeuvre et de la célébration de la création québécoise, a été perturbée en première partie de concert par les applaudissements d'une partie du public, lequel répondait positivement à la virtuosité du pianiste entre chaque prélude. Les responsables du festival avaient pourtant demandé d'applaudir uniquement entre les parties, donc à l'entracte et en fin de concert. Dès lors, l'une des conventions les plus solidement établies dans l'écoute de la musique classique en concert (c'est-à-dire le fait d'applaudir une fois l'oeuvre terminée) est devenue, d'un prélude à l'autre, la source d'une tension sociale au sein du public entre ceux qui voulaient applaudir et ceux qui revendiquaient le droit au silence. Finalement, le silence fut rétabli à partir du huitième prélude, mais au prix d'un recours à l'autorité qu'il importe de décrire avec précision pour en comprendre les incidences sur le concert, la portée culturelle et la dimension historique. Par le fait même, c'est la pratique d'écoute en concert qui est questionnée quant à ses finalités esthétiques. D’autant que dans le contexte festivalier où a été créée l'oeuvre de Dompierre, la posture générale d'écoute favorise une situation plus relâchée, où peuvent intervenir des formes de dissension entre les valeurs des uns et des autres. C'est la raison pour laquelle l'étude s'appuie sur la sociomusicologie, qui est la discipline la mieux placée pour analyser les conventions et les rituels au fondement de notre relation à la musique, tout en dévoilant le contenu historique des pratiques culturelles comme celle de l'écoute de la musique classique. 


\title{
Les applaudissements comme source de tension : Étude de la réception des 24 Préludes de François Dompierre lors de leur création au Festival de Lanaudière ${ }^{1}$
}

\author{
Danick Trottier \\ (Université du Québec à Montréal)
}

$\mathrm{L}$ a coutume en musique classique veut que lorsqu'une performance prend fin, les mélomanes applaudissent pour exprimer leur satisfaction et remercier les artistes; de même, lorsque les musiciens entrent en scène, les auditeurs applaudissement pour les accueillir. Le monde de la musique classique est structuré de telle façon que les applaudissements, qui permettent au public de communiquer son appréciation, constituent un geste normalisé dans la pratique du concert.

En ce sens, le fait d'applaudir à la fin d'une prestation correspond en tout point à ce que Howard S. Becker (1988, 64-88) entend par convention dans le fonctionnement des mondes de l'art ${ }^{2}$. Si Becker porte d'abord son attention aux conventions qui régissent la production des œuvres d'art, en l'occurrence les pratiques adoptées par les différents membres d'un monde de l'art et qui rendent ce monde efficace dans la division des tâches qui incombent à chacun, le sociologue américain fait aussi allusion au pôle de la réception, à la façon dont les spectateurs acquièrent une expérience en fréquentant les institutions culturelles. Le jeune auditeur qui assiste pour la première fois de sa vie à un spectacle imite ainsi ses voisins lorsqu'ils applaudissent à la fin d'une œuvre: le geste lui devient familier et il le reproduit à son tour.

Rien n'est plus normalisé qu'un concert de musique classique, alors que le concert rock apparaît comme l'expression la plus libre des réactions spontanées et sans inhibition. D'ailleurs, cette situation qui oppose les concerts classique et rock a conduit Antoine Hennion (1993, 324-326 et 335-339) à parler d' «empire de la musique» pour décrire le premier et de «scène primitive» pour décrire le second: les scènes de musique classique imposent une distance en plaçant l'auditeur dans une forme d'introspection pour maximiser l'écoute, tandis que les scènes de musique rock offrent une relation directe entre l'artiste et le public. Autrement dit, alors que la scène classique s'évertue à recréer un modèle de société fortement normalisé, où les désirs ne s'expriment qu'à des moments précis, fixés dans une pratique qui a subi l'épreuve du temps, la scène rock donne lieu à toutes sortes de réactions en déployant un modèle de société totalement relâché où s'expriment les désirs les plus variés. Le monde de la musique classique en situation de concert est à ce point normalisé que cette convention se vit sous forme d'autorité: applaudir au mauvais moment (par exemple entre les mouvements d'une symphonie) peut être perçu comme un écart de conduite.

Mais qu'arrive-t-il quand une convention n'est pas respectée? Dans la présente étude de nature sociomusicologique $^{3}$, une institution culturelle et un concert en particulier retiendront mon attention alors que j'analyserai les enjeux à la fois sociaux, esthétiques et historiques derrière la convention des applaudissements (et par extension la convention du silence en situation de concert). Il s'agit de la création des 24 Préludes, en forme de boogie et de bien d'autres choses encore... (dorénavant appelés 24 Préludes) de François Dompierre ${ }^{4}$ par le pianiste

\footnotetext{
Je tiens à remercier chaleureusement Claudine Caron et Jean Boivin pour leurs commentaires critiques et leurs suggestions de correction.

2 Becker définit ainsi ce qu'il entend par monde de l'art: «Un monde de l'art se compose de toutes les personnes dont les activités sont nécessaires à la production des œuvres bien particulières que ce monde-là (et d'autres éventuellement) définit comme de l'art.» $(1988,58)$.

3 La sociomusicologie en tant que façon particulière d'étudier l'objet musical se faufile dans les écrits musicologiques depuis un certain temps, mais sans avoir été systématisée. À titre d'exemple, dans la version française des conférences d'Adorno rapportées sous le titre d'Introduction à la sociologie de la musique (1994), ce dernier emploie à de nombreuses reprises le concept de «musico-sociologie» (ce qui est une variante de la sociomusicologie), tandis que John Shepherd (1982) titre un article paru dans le second volume de la revue Popular Music: «A Theorical Model for the Sociomusicological Analysis of Popular Musics». Simplement définie, la sociomusicologie est pratiquée par des musicologues qui ont recours à la fois aux outils de la musicologie et des sciences sociales pour étudier la musique à travers ses dimensions sociales, économiques et politiques.

4 François Dompierre est connu au Québec comme directeur musical, compositeur et orchestrateur, entre autres à travers ses collaborations avec des interprètes du milieu de la chanson, tels Pauline Julien et Félix Leclerc, ou des cinéastes, tels Denys Arcand et Michel Brault. Il a aussi écrit plusieurs œuvres dans un style néoclassique et postromantique, dont des concertos pour violon, piano et trombone ou Les Glorieux créés par l'Orchestre symphonique de Montréal et Kent Nagano en 2008.
} 
Alain Lefèvre ${ }^{5}$ le 14 juillet 2012 à l'amphithéâtre FernandLindsay, dans le cadre de la $35^{\mathrm{e}}$ édition du Festival de Lanaudière. Le récital entier était consacré à cette nouvelle œuvre (dont l'audition a été interrompue par une pause). Dans la première partie du concert, la création des Préludes a failli tourner au vinaigre en raison des applaudissements qui survenaient entre chaque prélude, certains mélomanes choisissant d'ignorer la consigne émise par Alex Benjamin, le directeur artistique du festival, au début du concert; celui-ci a en effet demandé d'applaudir uniquement entre les parties. Que se passe-t-il lorsqu'une convention faisant normalement autorité est malmenée, et ce, au point où le concert devient la manifestation d'une tension sociale et que l'attention des mélomanes en est affectée? Avant de décrire l'événement, quelques précisions s'imposent pour situer la présente étude et l'institution qu'est le Festival de Lanaudière.

Les résultats de recherche présentés ici découlent des travaux réalisés à l'été 2012 par l'équipe de sociomusicologie (équipe que je codirigeais avec Michel Duchesneau) de l'Observatoire interdisciplinaire de création et de recherche en musique (OICRM). Lors de cette édition du Festival de Lanaudière, j' ai réalisé un travail de terrain sous forme d'une première cueillette d'informations pour le projet intitulé «Développement de public en musique au Québec ${ }^{6}$ ». Il réunit des chercheurs (des musicologues mais aussi des universitaires rattachés à des disciplines connexes) dans le but notamment d'étudier le public mélomane au Québec et d'en décrire l'évolution au fil des décennies. Le projet vise des partenariats avec certaines institutions culturelles qui veulent bien se lancer dans l'aventure, ce qui est le cas du Festival de Lanaudière. La méthodologie s'appuyait essentiellement sur deux types d'observation: l'observation directe et l'observation participante - par moments, j'étais en retrait et j'observais les comportements du public, tandis qu'à d'autres moments je prenais place au sein du public. Dans le contexte du rituel de concert, il est à noter que l'observation en temps réel est la méthode d'investigation la plus appropriée lorsqu'il s'agit de jauger les impressions d'après-concert ${ }^{7}$.

Comme les prochaines lignes tenteront de le montrer, la réception d'un concert dépend du contexte particulier dans lequel celui-ci se déploie. Les conventions de concert, en Occident, nous ont été transmises par une tradition musicale fortement normalisée quant aux comportements à adopter en situation d'écoute. Dans ce contexte, il appert aussi nécessaire d'interroger la finalité de ces conventions.

\section{La musique classique en contexte festivalier}

Le Festival de Lanaudière se déroule en période estivale et offre une programmation qui s'étend de la première semaine de juillet à la première semaine d'août ${ }^{8}$. Des lieux différents accueillent les concerts. En semaine, le festival propose des concerts de musique de chambre ou des récitals dans les différentes églises de la région. Mais ce qui le distingue et contribue largement à sa réputation est l'Amphithéâtre Fernand-Lindsay (nommé ainsi suite au décès du père fondateur du festival en $2009^{9}$ ) situé à Joliette, où le festival s'est enraciné à compter de 1989: les festivaliers y sont conviés les vendredis (en soirée), samedis (en soirée) et dimanches (en après-midi) pour des concerts en plein air, présentés majoritairement par des orchestres, mais parfois par des ensembles de musique du monde (surtout le dimanche après-midi).

Le site est particulier en ce qu'il offre deux situations d'écoute distinctes ${ }^{10}$. Devant la scène se trouve le parterre, qui compte 2000 places et qui est entièrement recouvert (comme la scène d'ailleurs) mais non fermé sur les côtés ni à l'arrière. Derrière les places assises, la pelouse monte en pente et les festivaliers peuvent s'asseoir directement sur le sol ou sur une chaise s'ils en apportent une. La pelouse, qui peut accueillir 4000 personnes, est un espace social fascinant dans le cadre d'une situation de concert: dès l'ouverture des portes à 18 heures les vendredis et samedis (donc deux heures avant le concert), certains festivaliers

\footnotetext{
5 Alain Lefèvre poursuit une carrière de pianiste virtuose depuis les années 1990. Il se produit avec les orchestres du monde entier en présentant des concertos pour piano issus du long XIX ${ }^{\mathrm{e}}$ siècle, surtout postromantiques, à quoi s'ajoute sa défense de l'œuvre d'André Mathieu (1929-1968) qu'il a remis à l'honneur en interprétant ses concertos. Il cumule de nombreux prix depuis quelques années, par exemple celui de «Personnalité de l'année $2009 »$ lors du Gala Excellence organisé par La Presse et Radio-Canada.

6 Ce projet s'appuie sur un désir de renouveler, rajeunir et accroître le public qui fréquente les institutions musicales du Québec. Dans cette perspective, un espace plus expérimental est mis en place pour tester de nouveaux dispositifs et repenser la grammaire du concert.

7 Étant donné les réalités temporelles du concert, des méthodes comme le questionnaire ou l'entretien sont plus difficiles à appliquer dans une situation comme celle décrite ici, et cela pour deux raisons. Premièrement, on ne peut prédire à l'avance ce qui va survenir, rendant ainsi impossible la préparation d'un questionnaire; deuxièmement, à la fin d'un concert, le public se disperse rapidement, l'entretien devenant ainsi une course à obstacle avec un échantillon restreint.

8 Pour la petite histoire, le Festival de Lanaudière a pris forme en 1977 à l'initiative de son fondateur, le père Fernand Lindsay, et n'a cessé de croître en devenant l'un des festivals les plus importants au Québec (autant pour son rayonnement que pour sa notoriété), le tout se déroulant dans la région de Lanaudière (non loin de la métropole montréalaise).

9 Le clerc de Saint-Viateur Fernand Lindsay (1978-2009) a été à la fois homme de foi, directeur musical, chef d'orchestre, organiste et professeur. Il a fait carrière principalement à Joliette où il a développé le Festival de Lanaudière ainsi que le Camp Musical de Lanaudière.

10 Le lecteur peut avoir une idée de l'Amphithéâtre Fernand-Lindsay en visionnant un extrait de l'émission Sur les routes des Festivals consacrée au Festival de Lanaudière (Production Présence inc. 2012). Le site Internet du festival propose aussi une galerie de photographies renouvelée année après année (voir Festival de Lanaudière s.d.).
} 
prennent place sur la pelouse, généralement en famille ou entre amis, autour d'un repas.

C'est dire que dans ce lieu qu'est l'Amphithéâtre Fernand-Lindsay, la musique classique se transmet dans un cadre bucolique. L'harmonie est parfois parfaite, comme au moment où les oiseaux chantent leur refrain du soir, et parfois imparfaite, par exemple quand la météo n'est pas clémente. L'expérience du concert y est en soi particulière, suffisamment pour que le Festival de Lanaudière perdure depuis plus de 35 ans et soit reconnu comme le plus grand festival de musique classique au Canada ${ }^{11}$. En moyenne, de 2000 à 6000 personnes se réunissent pour y écouter un concert classique, ce qui montre l'ampleur de l'événement. Parmi les nombreux artistes et ensembles de calibre international qui s'y sont produits au cours des dernières années, on peut mentionner le Deutsche Kammerphilharmonie Bremen dirigé par Paavo Järvi et le Pittsburgh Symphony Orchestra et son chef Manfred Honeck.

Il importe à ce stade d'insister sur la démarcation relationnelle que créent, dans l'amphithéâtre, les deux espaces physiques quant à l'attention portée durant le concert. L'auditeur n'écoute pas avec la même attention s'il se trouve au parterre ou sur la pelouse: il suffit en tant que mélomane d'en faire l'expérience. Au parterre, la proximité de la scène rend l'expérience plus vivante, tandis que la pelouse, plus distante, place l'auditeur dans une position de détachement plus grande, même si cela ne gêne guère, normalement, une écoute attentive. Dans la partie gazonnée, il est plus facile de se lever pour avoir accès aux toilettes et on court davantage le risque d'être dérangés par les moustiques et les bruits provenant de la forêt environnante (c'est-à-dire les hurlements de chiens, les hydravions, etc.).

C'est dans ce cadre précis que se vit, concert après concert, une situation qui semble vouloir se généraliser à l'Amphithéâtre Fernand-Lindsay: les applaudissements surviennent après le premier mouvement d'une symphonie ou entre les différentes sections d'une œuvre, bref, dès que les musiciens arrêtent de jouer. Deux exemples survenus à l'été 2012 seront brièvement décrits avant d'en arriver à l'objet de la présente étude. La saison 2012 s'est ouverte le samedi 7 juillet par la prestation d'un orchestre et d'un chœur formés de musiciens rassemblés par le festival sous la direction du chef d'orchestre Jean-Marie Zeitouni ${ }^{12}$.
L'œuvre à l'honneur, précédée de deux ouvertures (la Fanfare for the Common Man de Copland et la Fanfare for the Uncommon Woman de Tower), était le Requiem de Berlioz. Ce dernier se composant de dix mouvements et le concert étant donné sans pause, une partie du public (plus de la moitié) a applaudi entre les différents mouvements (l'autre partie du public se contentant d'applaudir à la fin).

Le samedi 21 juillet, les invités étaient cette fois le Pittsburgh Symphony Orchestra, son chef Manfred Honeck et le violoncelliste Johannes Moser. Après une ouverture de Glinka, figurait au programme le Concerto pour violoncelle de Dvořák: si les applaudissements se sont fait entendre à la fin du premier mouvement (c'est-à-dire l' «Allegro»), le chef a choisi d'enchaîner le deuxième mouvement («Adagio ma non troppo») au troisième («Allegro moderato ») pour respecter la convention en jeu. C'est d'ailleurs un procédé qu'adoptent plusieurs chefs, par exemple Kent Nagano lorsqu'il dirige l'Orchestre symphonique de Montréal (OSM). Pour éviter que des applaudissements surviennent entre les mouvements d'une symphonie, le chef garde les mains tendues avec la baguette vers le haut, comme si la musique n'était pas encore terminée, ce qui lui permet d'enchaîner les mouvements. La deuxième partie du programme de l'orchestre de Pittsburgh était réservée à la Cinquième symphonie de Tchaikkovski. Fait assez étonnant, les festivaliers ont encore une fois applaudi après le premier mouvement («Adagio - Allegro con anima »), mais guère après les autres mouvements, d'où la nécessité de tenir compte des attentes des auditeurs selon le discours musical déployé - j’y reviendrai plus loin. Quant aux festivaliers qui respectaient la convention selon laquelle on n'applaudit pas entre les mouvements ou les parties d'une œuvre, certains (quoique peu nombreux) ont alors cherché à faire régner le silence par des «chuts » et autres gestes d'impatience.

\section{La création des 24 Préludes de François Dompierre par Alain Lefèvre}

L'événement à la base de la présente étude est cependant d'un autre ordre et montre qu'une situation peut dégénérer lorsqu'une convention bien établie n'est pas respectée. Le concert a eu lieu le samedi 14 juillet 2012 à 20 heures, toujours à l'Amphithéâtre Fernand-Lindsay de Joliette. Fait exceptionnel, les festivaliers étaient conviés à un récital de piano (ce sont plutôt des orchestres qui se produisent dans

\footnotetext{
11 Le Festival de Lanaudière, bien qu'original dans le contexte canadien, n'est pas unique en son genre. Il s'inscrit plutôt dans les festivals de musique classique qui ont vu le jour au cours des années 1970, où se manifeste une situation propice à la consommation, au marché de la musique et au tourisme culturel (Vlasselaer 2003, 1009-1031). En ce sens, le Festival de Lanaudière est typiquement nord-américain: il est né du désir de valoriser un lieu bucolique en contexte régional, ce qui explique en partie son succès. Aux États-Unis, la prolongation de la saison d'un orchestre symphonique tient lieu de festival, selon le modèle établi par l'Orchestre de Boston à Tanglewood. La situation est différente à Lanaudière puisqu'aucun orchestre symphonique n'y est directement rattaché. En ce sens, avec l'ouverture de son parterre, le Festival de Lanaudière partage beaucoup de similitudes avec le festival de Santa Fe au Nouveau-Mexique, fondé en 1957 par le chef d'orchestre John Crosby.

12 Il s'agit du jeune chef d'orchestre qui a succédé à Yuli Turovsky à la direction musicale et artistique de l'orchestre de chambre I Musici de Montréal.
} 
ce cadre), donné par un musicien bien connu au Québec: le pianiste Alain Lefèvre. Sa carrière très médiatisée ${ }^{13}$ et sa popularité auprès du grand public se sont construits notamment autour de la monumentalisation du compositeur et pianiste québécois André Mathieu ${ }^{14}$ (1929-1968), qu'il a permis par ailleurs de redécouvrir (Trottier 2010). Il faut dire qu'Alain Lefèvre jouit en outre d'un statut particulier au festival, puisqu'il en est l'ambassadeur artistique depuis 2011. Ainsi, lors du lancement médiatique de cette édition au début du mois de juillet, il a fait la promotion de son propre concert sur plusieurs tribunes en compagnie du directeur artistique Alex Benjamin.

L'événement était attendu depuis un certain temps puisque la création de l'œuvre de Dompierre était initialement prévue à l'été 2011. Elle a finalement été reportée en raison des différentes modifications demandées par Lefèvre, insatisfait de certains passages quant à l'écriture pianistique. Il faut préciser que les deux hommes sont amis et que les 24 Préludes ont été écrits spécifiquement pour le pianiste ${ }^{15}$. Le directeur artistique du festival, Alex Benjamin, a mené une entrevue avec Alain Lefèvre où ce dernier explique la genèse de l'œuvre et parle des difficultés qu'elle présente sur le plan technique ${ }^{16}$.

Ce qui ressort de cette vidéo et des autres entrevues accordées par Lefèvre dans les médias (voir par exemple Gingras 2012a), c'est la fébrilité du pianiste et une certaine inquiétude: si la virtuosité des différents préludes est un facteur non négligeable d'appréhension - le pianiste évoque le «million de notes à travailler» dans l'entrevue vidéo -, on doit aussi tenir compte des enjeux associés à cette création et de la réputation de l'artiste. Car l'idée de vendre le génie musical québécois reposait sur le succès de l'entreprise et donc, par extension, sur les épaules de Lefèvre.

Comme ce fut le cas à plusieurs reprises durant la période où il s'est consacré aux œuvres d'André Mathieu (voir Trottier 2011), le discours de Lefèvre a versé dans une certaine enflure verbale et une forme de nationalisme culturel. Le 6 juillet 2012, à l'émission Maintenant vous le savez! de la station de radio 98,5 où il était interviewé par le journaliste Benoît Dutrizac (voir Huss 2012), Lefèvre a affirmé que les 24 Préludes de Dompierre doivent être considérés comme «un chef-d'œuvre de la musique classique» et que ceux qui s'opposent à de tels projets artistiques s'adonnent à une forme de "Québec bashing», cela avant même que l'œuvre ait été créée! Nous sommes ici en présence d'un artiste qui cherche à influencer en amont la réception d'un événement, en opposant deux camps: ceux qui le suivront et ceux qui le critiqueront. Cette entrevue témoigne-t-elle de l'insécurité qu'a vécue le pianiste à la veille de la création des 24 Préludes? Le lendemain du récital (c'est-à-dire le dimanche 15 juillet 2012), en entrevue à l'émission matinale de Franco Nuovo, Dessine-moi un dimanche, de la Première Chaîne de Radio-Canada (Nuovo 2012), il a avoué qu'il était de plus en plus nerveux le jour du concert.

Cette nervosité a pu avoir une incidence sur la suite des événements. Le concert était ainsi organisé: les préludes numéros 1 à 13 ont été entendus dans la première partie du concert, puis les préludes numéros 14 à 24 après la pause. Juste avant que François Dompierre ne prenne la parole pour présenter brièvement son œuvre, en insistant notamment sur l'influence des musiques nord-américaines, dont le jazz, le directeur artistique du festival, Alex Benjamin, s'est lui aussi adressé au public. Après avoir insisté sur le fait que le festival était heureux d'accueillir cet événement et qu'il se faisait un devoir de mettre les compositeurs québécois à l'honneur, Benjamin a demandé, comme je l'ai mentionné plus haut, qu'on applaudisse «entre les parties ${ }^{17} »$. La foule avait donc été prévenue de la marche à suivre, à ceci près que la notion de parties a pu porter à confusion.Pour tout mélomane averti, la fin des parties est délimitée par l'intermission et la fin du concert, mais on peut penser qu'il n'en va pas nécessairement de même pour les mélomanes plus néophytes, d'autant que le programme de concert ne regroupait pas les préludes en parties, d'où la possibilité d'un malentendu. Dans ce contexte, il aurait été préférable de demander clairement au public d'applaudir avant la pause et à la fin du concert.

Ce qui devait se produire s'est produit: après le premier prélude, intitulé «Frénétique», le public a chaudement applaudi l'interprète, et ce public était présent autant à la pelouse qu'au parterre. La musique et le jeu du pianiste favorisaient incontestablement cet élan de spontanéité en faveur des applaudissements. Sans entrer dans une description détaillée de chacune des pièces, quelques

13 La médiatisation de sa carrière l'a transformé en véritable vedette du show business québécois. Il est invité à diverses tribunes médiatiques, comme ce fut le cas en mai 2010 lors de son passage à l'émission Tout le monde en parle dans la foulée de la sortie du film de Luc Dionne L'enfant prodige. À cette réalité s'ajoute sa participation à Espace Musique de la Société Radio-Canada à titre d'animateur, soit à la barre de l'émission Dans les carnets d'Alain Lefèvre le dimanche matin.

14 Après un semblant de pause dans la défense de l'œuvre de Mathieu, puisqu'il avait ralenti le nombre de pièces jouées tout en étant plus discret dans ce dossier, Lefèvre semble avoir repris le bâton du pèlerin au cours de l'année 2013. À titre d'exemple, le 9 décembre, il a interprété au mythique Carnegie Hall de New York le Concertino et le Concerto $n^{\circ} 4$ sous la baguette de JoAnn Falletta avec l'Orchestra of St Luke's. Nathalie Petrowski (2013) a rapporté dans le quotidien La Presse la justification historique derrière l'évènement et la fébrilité de Lefèvre.

15 L'œuvre a été enregistrée en juillet 2012 à l'église Saint-Benoît de Mirabel (Dompierre 2012c).

16 L'entrevue a été mise en ligne (Festival de Lanaudière 2012).

17 Alex Benjamin, après avoir apporté cette précision, a ajouté que Lefèvre accorderait une séance de signature à la fin du concert. 
éléments musicaux doivent être pris en compte. D'une part, les rythmes serrés et rapides, les formules arpégées en accélération et la profusion de notes exigeaient une grande virtuosité, ce qui a conduit le critique du Devoir Christophe Huss (2012) à écrire que l'œuvre «était inutilement complexifiée [sic] dans les moyens». D'autre part, le discours musical (phrasé, harmonie et rythme) était assez familier à tout auditeur de musique classique et de jazz. L'auditeur doté d'une certaine culture musicale n'était jamais complètement décontenancé et pouvait se retrouver à tout moment dans le continuum musical ${ }^{18}$. Dans les notes de programme, François Dompierre a décrit ainsi sa démarche:

L'auditeur attentif remarquera que ces préludes, pourtant conçus avec une notation et des matériaux traditionnels, font référence à la vie d'aujourd'hui. Leur construction, leur déroulement, les idées rythmiques qu'on y découvre, les modulations qu'on y rencontre, la manière dont elles sont amenées, n'ont la plupart du temps qu'un rapport lointain avec ce qu'on trouve dans la musique du temps passé. La mienne s'inspire quelquefois du jazz, des musiques populaires, du grand répertoire pianistique français. Elle est à la fois tout et rien de cela (Dompierre 2012b).

Enfin, les rythmes populaires et les formules mélodicorythmiques en ostinato étaient fortement exploités ${ }^{19}$, donnant aux différents préludes une fin souvent prévisible.

Cette prévisibilité apparaît fondamentale pour expliquer les applaudissements qui ont suivi les premiers préludes, d'autant que l'auditeur était confronté à des fonctions cadentielles fortement établies - le plus souvent des cadences parfaites ou des demi-cadences. Dans la mesure où, comme le décrit Leonard $\mathrm{B}$. Meyer dans son livre phare Emotion and Meaning in Music (1956; 2011 pour la traduction française), «sur un plan très général, l'attente est toujours en avance sur la musique, et crée un fond de tension diffus par rapport auquel les retards et autres "accidents" articulent la courbe des affects et créent du sens» (2011, 106), l'auditeur était non seulement en terrain connu quant à la forme et au langage de ces préludes, mais pouvait aussi anticiper les moments de clôture (au sens de closure en anglais). Le moment d'excitation propre à l'anticipation favorisait ainsi les applaudissements. En fait, le virtuose Alain Lefèvre a joué les premières pièces à une vitesse époustouflante et il n'est pas étonnant que le public ait réagi spontanément. Il s'agissait d'une réponse émotionnelle suscitée par l'émerveillement. L'expérience fortement acquise des styles musicaux, comme le propose Meyer $(2011,107)$, induit en outre une forme d'anticipation. À cet effet, il fallait voir le pianiste à l'œuvre pour rendre tangible cette dimension de la partition: chaque prélude se concluait par des gestes d'éclats comme s'il s'agissait de la fin d'un concerto pour piano. En ce sens, il était à prévoir qu'à cette virtuosité et cette profusion de notes et d'effets musicaux familiers le public allait répondre par des applaudissements. Les attentes lors de la création d'une nouvelle œuvre s'appuient nécessairement sur des références connues, plus précisément sur les «réactions d'habitude des auditeurs» (Meyer 2011, 107) issues de l'apprentissage. Ici, l'œuvre renvoyait directement aux effusions techniques du répertoire pianistique des XVIII ${ }^{\mathrm{e}}$ et $\mathrm{XIX}^{\mathrm{e}}$ siècles.

Les applaudissements sont ainsi survenus à la fin de chacun des premiers préludes, et on pouvait voir le visage de Lefèvre exprimer une certaine impatience. Il faut ajouter que le contexte de création n'était pas clément puisque l'artiste, en habit de concert, affrontait ce soir-là une température accablante d'environ 32 degrés Celsius en tenant compte du facteur humidex; le pianiste devait s'éponger le front entre chacun des morceaux. Toujours est-il qu'après le troisième prélude, le pianiste a mis son index devant sa bouche pour tenter d'imposer le silence. La tension devenait de plus en plus palpable: si une partie importante du public continuait d'applaudir après chaque prélude, une autre partie ripostait par des «chuts » et autres signes d'impatience.

Clairement, le public était alors fractionné en trois camps : ceux qui applaudissaient, en forte majorité; ceux qui imploraient les autres de se taire, en quantité moins importante; et ceux enfin qui étaient en mesure de sentir la tension. Après la cinquième pièce, les efforts des partisans pour le respect des conventions ont commencé à porter fruit puisque le public qui applaudissait Lefèvre diminuait en importance. Mais à la fin du sixième prélude, il restait toujours une masse critique voulant applaudir, et cela malgré la fronde des partisans du silence. C'est dire qu'un certain public croyait dur comme fer qu'il était non seulement acceptable, mais même respectueux d'applaudir après chacun des préludes. Il y avait donc méconnaissance de la convention ou un désir plus grand encore d'exprimer sa satisfaction.

\footnotetext{
18 L'écriture musicale de Dompierre se situe généralement dans une lignée néoclassique, voire postromantique; le langage contient des références tonales et puise dans les conventions stylistiques des XVIII et XIX ${ }^{e}$ siècles. L'attrait du compositeur pour la forme traditionnelle du concerto (divisé en mouvements) témoigne de cette réalité. Ce qui ressort le plus clairement de son style, c'est l'importance qu'il accorde au travail mélodique. Dans le texte «Musique et séduction » qu'il a écrit pour la revue Transposition (2012a), il résume quatre trucs pour susciter l'émotion chez l'auditeurspectateur: la répétition, la mélodie accrocheuse, la naïveté de l'inspiration et l'artifice descriptif. Ces quatre traits stylistiques ressortent de manière évidente à l'écoute des 24 Préludes.

19 Comme l'ont noté plusieurs observateurs dont Gingras (2012b) et Huss (2012), les influences de Maurice Ravel, George Gershwin, George Antheil et Conlon Nancarrow sont perceptibles à l'écoute des différents préludes.
} 


\section{Le recours à l'autorité ou comment faire régner le silence}

La tension sociale a atteint un paroxysme après le septième prélude alors que le camp du silence et celui des applaudissements se sont à nouveau affrontés. Une auditrice assise au parterre a crié, de manière très autoritaire: "Vous applaudirez À LA FIN ${ }^{20}$ ». Une partie du public s'est mise à rire, montrant ainsi, à mon avis, son incompréhension et son inconfort. L'apostrophe de l'auditrice, qui se faisait ainsi la porte-parole du camp du silence, a atteint son objectif: le silence s'est mis à régner entre les préludes, et cela jusqu'à la fin du concert, les auditeurs réservant dès lors leurs applaudissements pour la fin de la première partie du récital, le retour de l'artiste sur scène et la fin du concert.

Ce moment a marqué une rupture au cœur du concert, l'attention de tout l'auditoire ayant été perturbée par un événement sans lien avec la prestation artistique. À compter de cet instant, on pouvait sentir que l'intérêt pour l'œuvre avait diminué, ou qu'à tout le moins le public manquait de concentration. Plusieurs conversations durant l'intermission évoquaient ce moment précis: il y était notamment question du «culot de la dame», du «manque de respect» pour l'artiste. Chacun faisait valoir son opinion sur ce qui venait de se passer. Sans aller jusqu'à parler de gâchis, il reste que cette rupture dans le déroulement normal du concert a fait réagir de nombreux auditeurs. Tant et si bien que le critique de La Presse, Claude Gingras, a senti le besoin (contrairement à son homologue du Devoir) de relater ce qui s'était passé et de s'en féliciter, cautionnant ainsi l'attitude d'une partie du public:

On avait demandé aux spectateurs de ne pas applaudir après chaque pièce. Bien sûr, ils applaudirent quand même... jusqu'à la septième. Bravo à la dame qui s'écria alors: «Vous applaudirez à la fin!!» À partir de la huitième, ce fut, enfin, le plus beau silence... Cette dame devrait se voir offrir une admission à vie à la Maison symphonique! (2012b).

Il faut dire que Gingras fait des applaudissements un enjeu important ${ }^{21}$. Se donnant un rôle de chien de garde de la tradition classique, il n'a de cesse d'insister sur le fait qu'il faut applaudir à la fin des œuvres, suppliant même les institutions de prendre les mesures nécessaires pour faire respecter cette convention.

S'il se dégage de cet événement une forme de controverse sociale, c'est bien dans les propos de Gingras: le camp des auditeurs fidèles à la convention des applaudissements réservés au moment opportun s'efforce de la faire respecter en tant que droit fondamental de l'écoute en situation de concert. Toutefois, la controverse s'est exprimée à sens unique: le camp des auditeurs plus néophytes n'a pas été secondé par une prise de parole publique de la part de leaders pouvant défendre cette position. Reste qu'une forme de désaccord s'est produite ce soir-là, dimension essentielle de toute controverse sociale (voir Lilti 2007): se sont affrontés des applaudissements et des interventions parlées (c'est-à-dire les «chuts!») au moment où d'ordinaire règne le silence dans la continuité de la prestation de l'artiste.

Ainsi les auditeurs ont-ils vécu trois phases bien distinctes durant ce concert, lesquels peuvent être résumés comme suit: 1) une période de tension progressive; 2) un moment de désaccord dont la résultante fut un certain inconfort; et 3) une forme de controverse se manifestant par un certain relâchement de la concentration dans la suite du concert, prolongé par un bref écho de la controverse dans les jours qui ont suivi (c'est-à-dire la critique de Gingras). Si le concert n'a pas été interrompu par cet incident, il a été néanmoins perturbé, assez pour que l'attention du public ait été détournée, au moins temporairement, de l'œuvre ellemême, même si Lefèvre - on s'en doute! - fut ovationné après les parties (moments d'un parfait accord social!).

\section{Le concert comme lieu de contrôle}

Une convention comme celle des applaudissements montre que le concert de musique classique fait intervenir un modèle de société fortement contrôlée. Le concert est un «champ de bataille» perpétuel, pour reprendre l'expression de Peter Szendy $(2000,96)$. Il s'agit d'un lieu où «les stratégies conflictuelles de l'écoute» (Szendy 2000, 96) se donnent à voir. Écouter une ouvre classique en concert exige une disposition particulière pour s'assurer d'un environnement sonore optimal. Car le geste d'applaudir au moment opportun s'appuie aussi sur une autre convention, qui en est d'une certaine manière l'extension: le silence comme moment d'une écoute attentive, vouée exclusivement à l'œuvre. S'il ne faut pas manifester bruyamment son enthousiasme à tout moment, c'est aussi parce qu'il faut éviter de perturber le silence, lequel doit régner jusqu'à la fin de l'œuvre.

En ce sens, le rituel du concert classique s'appuie sur une recherche d'unité dans sa dimension collective. L'enjeu qui se dessine en filigrane est celui des relations entre les individus qui prennent part au rituel, le groupe entrant dans une forme de cohésion sociale eu égard à la temporalité qu'implique la situation d'écoute dans une

\footnotetext{
20 Je mets le « À LA FIN» en majuscule puisque la dame a appuyé et détaché chacun des trois mots.

21 Gingras revient souvent sur cette question dans ses critiques de concert. À titre d'exemple, lors du passage de l'Orchestre du Festival de Budapest et de son chef Ivan Fisher à la Maison symphonique le mardi 22 janvier 2013, Gingras précise que « ce concert très long [...] avait attiré une salle comble dont il faut, à la fois, admirer l'écoute parfaitement silencieuse et regretter les applaudissements qui surgirent automatiquement entre les mouvements des trois œuvres au programme» (2013).
} 
salle. Christopher Small justifie ainsi le concept de rituel appliqué au rassemblement musical: «La manière dont les personnes doivent être reliées les unes aux autres définit une communauté, de sorte que les rituels sont utilisés à la fois comme un acte d'affirmation de la communauté, comme un acte d'exploration et comme un acte de célébration ${ }^{22}$.» L'affirmation d'une communauté s'impose ici comme une idée maîtresse, et, en ce soir du 14 juillet 2012, l'enjeu principal était, à mon avis, l'affirmation de la communauté des auditeurs aguerris de musique classique cherchant à faire respecter les conventions qui la régissent.

$\mathrm{Si}$, comme le propose toujours Small $(1998,96)$, le rituel met en scène des gestes en dehors de la prise de parole, qu'il implique les sujets de manière émotionnelle et qu'il est un facteur de stabilité et de cohésion sociale, alors on peut mieux comprendre pourquoi les réactions ont été aussi vives et opposées au moment de la création des 24 Préludes de François Dompierre par Alain Lefèvre. Là où une partie du public s'est sentie menacée dans ce qui la définit comme auditoire de musique classique, l'autre partie cherchait à célébrer l'artiste, non pas tant en redéfinissant les paramètres du concert classique qu'en laissant libre cours à ses émotions face à la virtuosité de Lefèvre. L'identité de ceux qui ont l'habitude du concert classique, qui ont intégré les conventions et qui se font un devoir de les respecter, a été perturbée, d'où le besoin de rétablir une forme de cohésion sociale. Il y avait clairement pour ces habitués le camp des justes, soit ceux qui savent de quoi il retourne, et le camp de ceux qui ne savent pas.

Rappelons que le concert à l'Amphithéâtre FernandLindsay était caractérisé par une forte ambiguïté quant à ses finalités esthétiques. D'abord, un pianiste vedette allait créer une œuvre d'un compositeur connu dans un lieu normalement consacré à la musique orchestrale. Or, François Dompierre n'évolue pas dans les cercles de musique contemporaine et jouit d'une réputation où se croisent plusieurs genres musicaux, au premier rang desquels on retrouve la musique pop et la musique de film $^{23}$. Que venaient donc voir et entendre les auditeurs en cette soirée du 14 juillet 2012? Quel était le principal centre d'intérêt du concert? L'interprète ou l'œuvre? Plus l'interprète d'un récital est connu, plus l'unité se crée autour de sa personne, comme c'est le cas avec des pianistes tels Maurizio Pollini, Alfred Brendel ou Martha Argerich (voir Escale 2000, 233 -
234). Mais en ce soir du 14 juillet 2012, la justification du concert reposait officiellement sur l'œuvre de Dompierre présentée en création; l'évènement était donc placé sous le signe de la nouveauté. Cette ambiguïté, que l'on pourrait qualifier d'identitaire, explique aussi l'origine de la tension sociale. Et elle est aussi importante que le malentendu sémantique qui a pris forme à la suite de la demande initiale d'Alex Benjamin (c'est-à-dire les applaudissements après les parties).

Pour ceux à qui on avait vendu le concert en mettant plutôt de l'avant le charisme d'Alain Lefèvre, célébrer le pianiste à tout moment allait de soi. Mais pour ceux qui venaient assister au concert pour entendre la création d'une œuvre nouvelle, respecter les conventions allait aussi de soi. Alain Lefèvre jouit d'une place de choix dans le show business québécois, comme son rayonnement médiatique en atteste: il attire un public de masse ${ }^{24}$, dans lequel se trouvent forcément des néophytes prêts à applaudir ses exploits pianistiques. D'ailleurs, les «ho!» et autres signes d'étonnement ont jailli autant de la pelouse que du parterre à mesure qu'il enchaînait les notes en véritable virtuose. Les attentes esthétiques de l'auditeur n'étaient pas les mêmes selon que l'interprète ou la création - voire les deux justifiait sa présence au concert.

\section{La musique comme grandeur culturelle}

Un bref détour historique est nécessaire pour comprendre dans quelle mesure le rituel propre au concert classique a été normalisé par plus de deux cent ans d'histoire. Cette situation pointe en direction de la grandeur culturelle assignée à la musique classique par l'entremise d'une écoute attentive. Avant le XIX ${ }^{\mathrm{e}}$ siècle, en ce qui a trait à la performance musicale, l'amusement étant la catégorie qui définissait le plus souvent l'attitude à privilégier, ce que William Weber décrit ainsi : «Le puissant rôle de la musique comme amusement plaçait l'autorité quant aux questions de goût entre les mains du grand public, et non des connaisseurs ou d'une académie ${ }^{25} »$. En concomitance avec le développement de la musique instrumentale et la forte autonomisation que connaissent les pratiques artistiques à la fin $\mathrm{du} \mathrm{XVIII}^{\mathrm{e}}$ siècle, petit à petit prend forme l'idéologie de la musique sérieuse, qui vient redéfinir les formes d'écoute et l'attitude générale à l'égard de la musique qu'on qualifie aujourd'hui de classique. Cette idéologie de la musique sérieuse s'est sédimentée en partie

\footnotetext{
22 «How people ought to relate to one another, of course, define a community, so rituals are used both as an act of affirmation of community ("This is who we are"), as an act of exploration (to try on identities to see who we think we are), and as act of celebration (to rejoice in the knowledge of an identity not only possessed but also shared with others).» (Small 1998, 95). C'est moi qui traduis.

23 Lui aussi rejoint un large public par l'animation radiophonique, soit à la barre de l'émission Les détours de Dompierre le dimanche à Espace Musique de Radio-Canada.

24 C'est un fait souligné par plusieurs critiques et qu'on observe année après année lors des concerts donnés par Lefèvre dans les institutions musicales québécoises. Dans le cadre du Festival de Lanaudière, un concert avec Lefèvre génère automatiquement plus de 4000 entrées au guichet.

25 «The powerful role of music as amusement put authority over taste into the hands of the general public, not connoissseurs or an academy.» (Weber 2008, 194). C'est moi qui traduis.
} 
autour de la figure de Beethoven dans la Vienne des années 1790, comme l'a montré Tia DeNora dans son livre consacré à la construction de la grandeur culturelle de ce compositeur (1998). Sans réécrire ici une histoire largement documentée, par exemple par Carl Dahlhaus (1989), je préciserai que le modèle beethovénien reconfigure les valeurs musicales de telle sorte que l'écoute attentive de l'œuvre devient une manière d'en saisir le sens. L'œuvre, en jouissant d'un nouveau statut (c'est-à-dire l'idéal de la Werktreue ${ }^{26}$ ), est placée au centre de l'attention quant au déchiffrement des intentions du compositeur.

C'est la raison pour laquelle chaque membre de l'auditoire doit désormais se réfugier dans le silence le plus complet eu égard au contenu de l'œuvre, favorisant ainsi une sorte de confrontation à la fois spirituelle et herméneutique quant au message qu'elle porte. Par exemple, dans l'Allemagne des années 1770 à 1848 s'impose, comme l'a montré David Gramit (2002), un nouveau paradigme culturel et politique: par le truchement de la musique on éduque le peuple à la culture. Mais dans la mesure où la culture se confond désormais avec la musique (celle-ci étant l'art placé au sommet des valeurs sociales et culturelles), être cultivé signifie posséder des connaissances musicales. La musique acquiert alors un prestige social sans précédent, lequel découle de l'idéologie de la musique sérieuse qui s'impose à Berlin, à Vienne, puis dans l'Europe du XIx ${ }^{\mathrm{e}}$ siècle, et dont l'ascension viennoise de Beethoven est l'exemple par excellence dans les années 1790. En d'autres termes, les élites et les acteurs du milieu musical cherchent à imposer un goût particulier, celui pour la musique sérieuse où priment les formes de profondeur que mettent en scène les musiciens. Le baron Van Swieten (1733-1803), fidèle supporteur de Beethoven et que l'on surnomme à l'époque le «patriarche de la musique» (voir DeNora 1998, 56-58), œuvre farouchement pour l'établissement des nouvelles conventions propres à cette musique en imposant auprès du public le silence et un ethos de la gravité par lequel jaillit l'autorité culturelle tant recherchée.

Mais pourquoi a-t-on voulu éduquer les mélomanes du XIX siècle? Dans la mesure où le concert se démocratise dans une économie de marché et s'ouvre alors à un nouveau public, il faut répandre la nouvelle identité de la musique classique. Cette identité s'appuie sur des rituels dont l'efficacité permettra une meilleure cohésion de la communauté des mélomanes et une meilleure préservation des valeurs de prestige que suppose l'idéologie de la musique sérieuse. La réception musicale étant définie de manière verticale en partant de l'autorité du compositeur, la performance de l'œuvre doit être sécurisée en tant que moment singulier et significatif. Par exemple, Eduard Krüger (1807-1885), critique musical allemand, affirme en 1839 qu'un certain public pose problème dans les centres urbains et identifie même un «cancer du dilettantisme» (voir Gramit, 2002, 148). Tout comme le fait de nos jours Claude Gingras, des critiques influents du XIX ${ }^{\mathrm{e}}$ siècle comme A.B. Marx (17951866) insistent sur le fait qu'un public dilettante nuit au rituel de concert.

C'est dans ce contexte que deux catégories d'auditeurs vont être identifiées par les commentateurs du XIX siècle, soit d'un côté les auditeurs cultivés, de l'autre une catégorie plus fourre-tout incluant les dilettantes et les incultes, bref tous ceux qui ne comprennent pas la musique classique. Autrement dit, une catégorie d'auditeurs se définit par rapport au savoir qu'elle possède et, à coup d'autorité, circonscrit une autre catégorie d'auditeurs en lui imposant les conventions à la base de son identité d'auditeur. Cette situation montre comment le concert de musique classique fonctionne sur la base d'une idéologie qui s'est imposée comme tradition et qui vise à créer de l'unité sociale derrière des considérations culturelles fortement prescriptives.

Cette pratique de l'autorité verticale perdure depuis la mise en place de l'idéologie de la musique sérieuse et définit en partie, sinon en totalité, l'identité de la communauté des auditeurs de musique classique, d'où les réactions très vives lorsque ses fondements sont ébranlés par un public qui ne voit pas en quoi le fait d'applaudir à chaque fois que l'artiste arrête de jouer constitue un méfait. Pourtant, les prouesses techniques encouragées par la musique classique à travers l'étalage de la virtuosité ont incité de tout temps l'auditeur à répondre de manière émotionnelle à l'émerveillement (voir Deaville 2003).

\section{Conclusion}

L'idée de «malentendu culturel», qu'Emmanuel Pedler (2004) a théorisée pour analyser le fossé qui peut se creuser entre l'offre culturelle et les attentes du public au sein des institutions, est la face cachée de la tension sociale qui s'est manifestée en cette soirée du 14 juillet 2012. De fait, une partie du public, non négligeable, était simplement venue écouter Alain Lefèvre, et les 24 Préludes ne constituaient pas pour elle une entité globale; une autre partie du public, habituée au concert classique, venait découvrir une œuvre nouvelle et écouter Alain Lefèvre, et pour elle la structure du concert (c'est-à-dire l'œuvre formant un tout) avait un sens. C'est la raison pour laquelle le concert de musique classique reste le lieu par excellence pour faire respecter la tradition et les conventions rattachées à cette musique.

\footnotetext{
26 Lydia Goehr $(2007,205-242)$ utilise le concept de « Beethoven Paradigm» pour théoriser la nouvelle autorité culturelle qu'assument les compositeurs, ainsi que les nouvelles valeurs culturelles et herméneutiques assignées à l'œuvre en tant que dévoilement d'une vérité qui la dépasse.
} 
À cela s'ajoute l'enjeu du lieu où le concert a pris place. Comme le précise à nouveau Emmanuel Pedler dans son étude sur la salle de concert:

Au plus général, c'est l'identité singulière des institutions musicales étudiées qu'il est important d'évaluer. Si les prémisses de notre raisonnement sont valides, on doit s'attendre à la fois à de grandes variations d'un espace à un autre et à des tensions et des dissensions entre les valeurs qui sont mobilisées par les acteurs en présence $(2004,141)$.

L'Amphithéâtre Fernand-Lindsay n'est pas un lieu de concert comme les autres puisqu'il peut accueillir jusqu'à 6000 festivaliers, qui se répartissent entre deux espaces d'écoute distincts, comme je l'ai souligné plus haut. La musique classique s'y transmet dans un cadre bucolique désenclavé: il s'agit d'une perspective ouverte qui offre une forme d'écoute tout aussi ouverte. Dans ce contexte, il y a bel et bien «dissensions entre les valeurs qui sont mobilisées par les acteurs » (Pedler 2004, 141), une partie d'entre eux étant selon toute probabilité des mélomanes assidus qui cherchent à retrouver le cadre conventionnel de la salle classique, alors qu'une autre cherche à maximiser son expérience d'écoute dans un cadre acoustique qui déroge des habitudes. Il n'est pas étonnant que dans ce contexte puissent sourdre des tensions sociales.

En terminant, il est à se demander si l'autorité culturelle du concert classique et la rigidité de certaines conventions ne doivent pas faire l'objet d'une remise en question. À cet effet, le pianiste Emmanuel Ax rappelle que les applaudissements sont une réponse émotionnelle à la musique et invite la communauté des mélomanes à faire preuve d'une plus grande tolérance quant aux conventions en vigueur ${ }^{27}$ (Ax 2008).

En général, loin de cet arrêt sur image qui donne à voir un désaccord social, le concert classique montre aussi le public, qu'il soit assidu ou occasionnel, capable de s'adapter à la situation. C'est dire que le concert est aussi un espace de cheminement réflexif alimenté par une situation fortement relationnelle. D'ordinaire, à moins de vouloir jouer le rôle de chahuteur, l'auditeur cherche à se fondre dans la dimension collective de l'événement en laissant toute la place à la transmission de l'œuvre. Le mélomane est alors appelé à faire des compromis en acceptant les rituels propres au concert de musique classique. Cette situation relationnelle, où chacun doit tenir compte des autres, explique en partie la capacité des auditeurs à s'adapter à la situation et le fait qu'en définitive, le concert classique, au Québec comme ailleurs, se déroule généralement sans heurt jusqu'à la manifestation de l'appréciation esthétique située, faut-il le rappeler?, à la fin de l'œuvre.

\section{RÉFÉRENCES}

Adorno, Theodor (1994). Introduction à la sociologie de la musique, Genève, Contrechamps. Traduit de l'allemand par Vincent Barras et Carlo Russi.

Ax, Emmanuel (2008). «When to Applaud», dans Emmanuel Ax's Official Blog, 14 novembre, http:// emanuelax.wordpress.com/, consulté le 25 octobre 2013.

Ax, Emmanuel (2009). "A Few More Thoughts on Applause: Why Can We Interrupt at the Met», dans Emmanuel Ax's Official Blog, 3 mars, http://emanuelax. wordpress.com/, consulté le 25 octobre 2013.

BeCKer, Howard S. (1988). Les mondes de l'art, Paris, Flammarion.

Dahlaus, Carl (1989). Nineteenth-Century Music, Berkeley, University of California Press.

Deaville, James (2006). "L'image du virtuose aux XVIII ${ }^{\mathrm{e}}$ et $\mathrm{XIX}^{\mathrm{e}}$ siècles: De Tartini à Paganini, et de Bach à Liszt», dans Jean-Jacques Nattiez (dir.), Musiques: Une encyclopédie pour le XXI siècle, Arles/Paris, Actes Sud/Cité de la musique, vol. 4 «Histoires des musiques européennes », p. 759-780.

DeNora, Tia (1998). Beethoven et la construction du génie, Paris, Fayard.

Dompierre, François (2012a). «Musique et séduction», Transposition: Musique et sciences sociales, $\mathrm{n}^{\circ} 2$, mai, http://transposition-revue.org/Musique-et-seduction, consulté le 25 octobre 2013.

Dompierre, François (2012b). «Notes de programme», Festival de Lanaudière, 14 juillet.

Dompierre, François (2012c). 24 préludes, en forme de boogie et de bien d'autres choses encore... Alain Lefèvre, piano, Analekta, AN 2 9292-3, 2 disques compacts (Dompierre: 24 Préludes).

EsCAL, Françoise (2000). «Le concert: Approche esthétique», dans Françoise Escal et François Nicolas, Le concert: Enjeux, fonctions, modalités, Paris, L'Harmattan, p. 219-253.

Festival de Lanaudière (s.d.). Le Festival de Lanaudière, http://lanaudiere.org/, consulté le 25 octobre 2013.

Festival de Lanaudière (2012). «Alain Lefèvre au sujet des Préludes de Dompierre», dans YouTube.ca, http://www. youtube.com/watch? $\mathrm{v}=\mathrm{e}-\mathrm{NjaoPO} 6 \mathrm{IM}$, consulté le 25 octobre 2013.

27 L'enjeu des applaudissements est discuté de manière fréquente sur les blogues des musiciens et des critiques musicaux. Le court texte d'Emmanuel Ax, intitulé «When to Applaud» (Ax 2008), a été suivi par un second intitulé «A Few More Thoughts on Applause: Why Can We Interrupt at the Met» (Ax 2009). 
Gingras, Claude (2012a). "Alain Lefèvre s'attaque à l'œuvre "monstrueuse" de Dompierre», dans LaPresse. ca, 14 juillet, http://www.lapresse.ca/arts/musique/ musique-classique/201207/13/01-4543481-alainlefevre-sattaque-a-loeuvre-monstrueuse-de-dompierre. php, consulté le 25 octobre 2013.

Gingras, Claude (2012b). «Dompierre et Lefèvre: Le triomphe», dans LaPresse.ca, http://www.lapresse. $\mathrm{ca} /$ arts/musique/musique-classique/201207/15/014543761-dompierre-et-lefevre-le-triomphe.php, consulté le 25 octobre 2013.

Gingras, Claude (2013). «Budapest: Pour Rachmaninov», dans LaPresse.ca, 23 janvier, http://www.lapresse. $\mathrm{ca} /$ arts/musique/musique-classique/201301/23/014614233-budapest-pour-rachmaninov.php, consulté le 25 octobre 2013.

Goenr, Lydia (2007). The Imaginery Museum of Musical Works: An Essay in the Philosophy of Music, Oxford, Oxford University Press. Édition revisée.

Gramit, David (2002). Cultivating Music: The Aspirations, Interests, and Limits of German Musical Culture, 17701848, Berkeley, University of California Press.

Hennion, Antoine (1993). La passion musicale: Une sociologie de la médiation, Paris, Métailié.

Huss, Christophe (2012). "Festival de Lanaudière Profus!», Le Devoir, 16 juillet.

LiLTi, Antoine (2007). «Querelles et controverses: Les formes du désaccord intellectuel à l'époque moderne», Mil neuf cent: Revue d'histoire intellectuelle, $\mathrm{n}^{\circ} 25$, p. 13-28.

Meyer, Leonard B. (2011), Émotion et signification en musique, Paris, Actes Sud, 2011. Traduit de l'anglais par Catherine Delaruelle.

Nuovo, Franco, animateur (2012). «François Dompierre au bout des doigts d'Alain Lefèvre» dans Dessinemoi un dimanche, 15 juillet, http://ici.radio-canada. ca/emissions/dessine_moi_un_dimanche/2013-2014/ chronique.asp?idChronique $=232871$, consulté le 25 octobre 2013. Entrevue avec Alain Lefèvre réalisée sur les ondes de la Première Chaîne de Radio-Canada.

PedLER, Emmanuel (2004). «Entendement musical et malentendu culturel: Le concert comme lieu de confrontation symbolique», Sociologie et Sociétés, vol. 26, $\mathrm{n}^{\circ} 1$, printemps, p. 127-144.

Petrowski, Nathalie (2013). «Faire revivre André Mathieu à Carnegie Hall», dans LaPresse.ca, 10 décembre, http://www.lapresse.ca/arts/musique/musiqueclassique/201312/11/01-4719835-lefevre-et-mathieufont-vibrer-carnegie-hall.php, consulté le 10 décembre 2013.
Production Présence inc. (2012). «Festival de musique classique de Lanaudière Québec», dans YouTube.ca, http://www.youtube.com/watch?v=yDv3D7vvy8Y, consulté le 25 octobre 2013. Extrait de l'émission Sur la route des Festivals au Festival de Lanaudière, Joliette, Québec présentée par TV5.

SHEPHERD, John (1982). «A Theorical Model for the Sociomusicological Analysis of Popular Musics», Popular Music, vol. 2, p. 145-177.

Small, Christopher (1998). Musiking: The Meanings of Performing and Listening, Hanover, Wesleyan University Press.

Szendy, Peter (2000). «L'art de la claque, ou: s'écouter écouter au concert»dans Françoise Escal et François Nicolas (dir.), Le concert: Enjeux, fonctions, modalités, Paris, L'Harmattan, p. 93-110.

Trottier, Danick (2010). «Monumentaliser la culture québécoise : Qu'aurait pensé Nietzsche de la canonisation d'André Mathieu? », dans Le Devoir, 29 mai, http://www. ledevoir.com/culture/musique/289826/monumentaliserla-culture-quebecoise, consulté le 25 octobre 2013.

Trottier, Danick (2011). "André Mathieu: Encore bien des questions... Georges Nicholson. André Mathieu: Biographie, précédée d'un entretien avec Alain Lefèvre », Les Cahiers de la Société québécoise de recherche en musique, vol. 12, n 1, p. 121-132. Essai recension.

Vlasselaer, Jean-Jacques Van (2003). «Les festivals de musique classique et d'opéra», dans Jean-Jacques Nattiez (dir.), Musiques: Une encyclopédie pour le XXI siècle, Arles/Paris, Actes Sud/Cité de la musique, vol. 1 «Musiques du XX $X^{\mathrm{e}}$ siècle», p. 1009-1031.

Weber, William (2008). The Great Transformation of Musical Taste: Concert Programming from Haydn to Brahms, Cambridge, Cambridge University Press. 